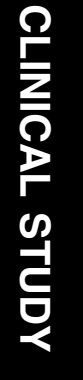

${ }^{1}$ Department of Ophthalmology, Graduate School of Medicine, University of Tokyo, Tokyo, Japan

${ }^{2}$ Department of Rehabilitation, Orthoptics and Visual Science Course, School of Allied Health Sciences, Kitasato University, Kanagawa, Japan

${ }^{3}$ Department of Ophthalmology, Tajimi Municipal Hospital, Gifu, Japan

${ }^{4}$ The Second Department of Ophthalmology, Toho University School of Medicine, Tokyo, Japan

Correspondence: A Tomidokoro, Department of Ophthalmology, Graduate School of Medicine,

University of Tokyo, 07-03-2001 Hongo, Bunkyo-ku,

Tokyo, 113-8655, Japan

Tel: + 8133815 5411;

Fax: + 81338170798 .

E-mail: tomidokoro-tky@ umin.ac.jp

Received: 15 May 2008 Accepted in revised form: 14 November 2008 Published online: 12 December 2008

This study does not include any duplicated information with other publications or submissions Commercial/proprietary interest: None

\title{
Atypical retardation pattern in measurements of scanning laser polarimetry and its relating factors
}

\begin{abstract}
Purpose To assess the prevalence of atypical retardation pattern (ARP) in scanning laser polarimetry (SLP) with variable corneal compensation (VCC) and enhanced corneal compensation (ECC), and to evaluate the factors relating to typical scan score (TSS). Methods Measurements of SLP-VCC, SLP-ECC, and the Humphrey field analyzer (HFA) were performed in 105 normal subjects and 82 open-angle glaucoma (OAG) patients. ARP was defined as an SLP image with TSS $<80$.
\end{abstract}

Results Prevalence of ARP with SLP-VCC was 13.3 and $14.6 \%$ in normal and OAG eyes, respectively, and that with SLP-ECC $(2.9 \%$ and $1.2 \%)$ was lower than SLP-VCC $(P<0.009)$.

TSS with SLP-VCC was significantly correlated with age (partial correlation coefficient $(\mathrm{PCC})=-0.22, P=0.003)$ and refractive error $(\mathrm{PCC}=0.26, P<0.001)$ after adjusting for each other. TSS with SLP-ECC was significantly correlated with neither age nor refractive error $(\mathrm{PCC}=-0.02, P=0.788$; $P C C=0.10, P=0.177$, respectively). In OAG eyes, mean deviation (MD) of HFA was significantly correlated with TSS with SLP-VCC and SLP-ECC (PCC $=0.35$, $P=0.001 ; P C C=0.23, P=0.039$, respectively). In SLP-VCC, MD was significantly correlated with retinal nerve fibrr layer (RNFL) thickness only after excluding eyes with ARP $(P<0.001)$. Conclusions ARP in SLP-VCC measurements was found in more than $10 \%$ of normal or glaucomatous eyes, and TSS was significantly lower in older subjects, more myopic eyes, or eyes with more advanced glaucomatous damage. The presence of ARP disturbs the accuracy of RNFL thickness measurement by SLP-VCC. In SLP-ECC
M Yanagisawa ${ }^{1,2}$, A Tomidokoro', H Saito ${ }^{1,3}$, C Mayama', M Aihara', G Tomita ${ }^{4}$, N Shoji ${ }^{2}$ and M Araie'

measurements, prevalence of ARP was considerably lower and TSS was not affected by age or refractive error, suggesting the advantages of ECC in clinical practices. Eye (2009) 23, 1796-1801; doi:10.1038/eye.2008.365; published online 12 December 2008

Keywords: scanning laser polarimetry; glaucoma; retinal nerve fibre layer thickness

\section{Introduction}

In glaucoma, structural changes in the optic nerve and the retinal nerve fibre layers (RNFL) are believed to precede functional changes found with standard automated perimetry. ${ }^{1-5}$ Therefore, sensitive measurements of RNFL thickness should be of clinical importance to detect early changes in glaucoma and to evaluate the progression of the disease from its initial stage.

In measurements, scanning laser polarimetry (SLP, GDx; Carl Zeiss Meditec, Dublin, CA, USA), atypical retardation (ie, birefringence) pattern (ARP) is sometimes found and the presence of ARP often makes it difficult to properly evaluate the SLP results, ${ }^{6-16}$ resulting in poorer performance in glaucoma diagnosis. ${ }^{11-14,16}$ ARP is a pattern of retardation, which do not match the histological distribution of RNFL thickness with thicker RNFL in the superior and inferior regions compared with the temporal and nasal regions. Recently, some clinical aspects of ARP have been reported, including the prevalence of ARP in normal and glaucomatous eyes ${ }^{9,13,16}$ and the impact of ARP on the performance of glaucoma diagnosis. ${ }^{11-14}$

The enhanced corneal compensation (ECC) has been developed as an alternative algorithm 
to the variable corneal compensation (VCC) method to improve the signal-to-noise ratio and eliminate artefacts associated with ARP. ${ }^{9,10,17,18}$ In the ECC system, a predetermined birefringence is introduced to the raw birefringence signal to shift it into a more sensitive region of the SLP detector. ${ }^{17}$ The amount of birefringence bias is determined using the birefringence pattern of the macular region, and then is mathematically removed point by point from the total birefringence pattern of the VCC to improve the signal and obtain a retardation pattern of the RNFL with less noise. ${ }^{17}$ It has been reported that the prevalence of ARP in ECC measurements is considerably lower than it is in VCC measurements. ${ }^{10,11,13,14}$ Bagga et al $^{8}$ reported that the presence of ARP in VCC measurements was associated with older patient age. However, the association of ARP with systemic or ocular factors in ECC measurements have not been fully understood, although it is empirically known that myopic eyes more commonly show ARP. ${ }^{9}$ The aim of this study is to evaluate the prevalence of ARP found in the measurements of VCC and ECC in normal and open-angle glaucoma (OAG) eyes and to investigate the factors relating to the presence of ARP in the both algorithms.

\section{Patients and methods}

All studies described below were carried out at the Department of Ophthalmology, Graduate School of Medicine, University of Tokyo, after obtaining a written informed consent from the subjects or patients. The studies were approved by the institutional review board and observed the declaration of Helsinki.

The SLP-ECC is a newly introduced algorithm, alternative to the VCC algorithm, without any alteration in the hardware of the currently used GDx VCC system. The principle of the ECC algorithm has been described in detail elsewhere $\mathrm{e}^{9,10,17,18}$ and is briefly summarized below. In both the VCC and ECC algorithms, an SLP image of the macula is first obtained to decide eye-specific anterior segment polarization. When measuring the RNFL thickness with the VCC algorithm, the magnitude of the corneal compensator included in the hardware is set to the same value as the anterior ocular segment retardance, and the slow axis is set to be perpendicular to the slow axis of the anterior ocular segment. As a result, the anterior segment polarization can be eliminated and the retardance of the RNFL is typically estimated in the range of $0-90 \mathrm{~nm}$.

In the ECC algorithm, after deciding the anterior segment polarization in each eye, the corneal compensator is set to make the combination of the polarization of the anterior segment and that of the compensator close to $55 \mathrm{~nm}$ with a vertical slow axis.
Consequently, the combined polarization signal originating from the RNFL and the anterior segment is enhanced to the range of $30-130 \mathrm{~nm}$, shifting the signal into a more sensitive region of the SLP detector. Finally, the predetermined polarization $(55 \mathrm{~nm}$ with a vertical axis) is removed from the obtained signal to determine the true RNFL retardance. With the ECC algorithm, the signal-to-noise ratio is increased by the enhancement of polarization signal, resulting in improvements on RNFL measurement in eyes with considerable noise interference originating from optical (eg, stray light, scleral reflection) or electronic (eg, noise, digitization error) error. ${ }^{17}$ For each measurement of SLP-VCC or ECC, typical scan score (TSS) ${ }^{9,10,18,19}$ was determined to evaluate the typicality of the polarimetry images. An image with ARP was defined as that with a TSS lower than $80 .{ }^{9,14}$

In this study, SLP measurements with VCC and ECC were performed in 105 ophthalmologically normal volunteers recruited from a large register for obtaining normal medical data and 82 consecutive patients with OAG from the outpatient clinic at Tokyo University Hospital between March 2005 and December 2005. Ophthalmologically normal eyes had no history of ocular diseases, intraocular pressure of $21 \mathrm{mmHg}$ or lower by Goldmann applanation tonometry, normal optic disc appearance based on clinical stereoscopic examination, and reliable and normal results of the Humphrey field analyzer (HFA) 24-2 Swedish interactive testing algorithm (SITA) standard strategy (Carl Zeiss Meditec Inc., Dublin, CA, USA). The results of the HFA were judged as reliable when fixation loss was $<20 \%$ and false-positive/negative error was $<33 \%$. A visual field result was concluded as normal when it showed neither glaucomatous abnormalities according to the criteria described below nor other pathological findings. OAG was diagnosed based on normal open angles, typical glaucomatous optic disc appearances, VFD corresponding to the optic disc findings evaluated with reliable HFA results with 30-2 SITA standard strategy, the absence of apparent pale colour of the optic disc, and the absence of any contributing ocular or specific systemic disorders. The visual field was judged to be abnormal according to the criteria described by Anderson and Patella. ${ }^{20}$

Eyes with refractive error (spherical equivalent) exceeding $\pm 8 \mathrm{D}$, eyes in which reliable SLP measurements with the VCC or ECC algorithms (quality score $=\geqslant 8$ ) could not be obtained, eyes with ocular diseases except OAG and slight cataract, eyes having the history of intraocular surgeries, and eyes with peripapillary atrophy situated within the measurement circle (diameter $3.2 \mathrm{~mm}$ ) of the SLP measurement were not included in this study. If both right and left eyes fulfilled the inclusion criteria, the data of right eye were used in the following analyses. 
SLP-VCC and -ECC measurements were performed on one SLP machine by switching the methods' software within the machine by an experienced examiner (MY). In every subject, VCC measurements were performed first and ECC measurements followed. All scans were checked for quality and scans with a $Q$ score lower than 8 were excluded from the study. The same measurement ring was used throughout the study, and the automatic disc edge identification was checked for each scan and manually adjusted when necessary. The SLP parameters investigated in this study were ellipse (TSNIT average), superior, and inferior averages, TSNIT SD, and nerve Fibre index (NFI). TSS ${ }^{16-18}$ was also obtained to evaluate the typicality of the polarimetry images. HFA measurements were obtained within 3 months from the SLP measurements.

\section{Results}

A total of 105 eyes of 105 normal subjects and 82 eyes of 82 OAG patients was included in this study. OAG patients were older than the normal subjects ( $59.1 \pm 8.5$ vs $50.6 \pm 15.0$ years, mean $\pm \mathrm{SD}, P<0.001$, unpaired $t$-test) and had more myopic refractive error $(-2.3 \pm 2.5$ vs $-1.2 \pm 2.3 \mathrm{D}, P=0.005)$. In normal and OAG groups, mean deviation (MD) of the HFA averaged $-0.72 \pm 1.2$ and $-3.3 \pm 4.1(P<0.001)$ and SD was $1.6 \pm 0.5$ and $5.4 \pm 4.3 \mathrm{~dB}(P<0.001)$, respectively. Table 1 summarizes the parameters of SLP-VCC and -ECC in the normal and OAG eyes. In the 105 normal eyes, TSNIT average and superior average were significantly greater in VCC than in ECC $(P<0.001, P=0.009$, respectively, paired $t$-test), whereas TSS, inferior average, and TSNIT SD were smaller in VCC (all $P<0.001$ ). In $82 \mathrm{OAG}$ eyes, similar trends were also found.

The prevalence of ARP (TSS $<80$ ) is shown in Table 2. The prevalence of ARP was significantly higher in VCC measurements than in ECC in normal and OAG groups ( $P=0.009$ and 0.002 , respectively, Fisher's exact test), although it was not significantly different between normal and OAG eyes for both VCC and ECC after adjusting for age and refractive error $(P=0.803,0.513$, respectively, Mantel-Haenszel test). In VCC measurements, age was not significantly different (53.9 \pm 12.9 vs $56.8 \pm 15.3$ years, $P=0.373$, unpaired $t$-test), whereas refractive error significantly more myopic in eyes with ARP than those without it $(-3.1 \pm 2.5$ vs $-1.5 \pm 2.4 \mathrm{D}, P=0.003$ ) between the 26 (14 normal and 12 OAG) eyes with ARP and the 161 (91 normal and 70 OAG) eyes without ARP. These comparisons were not done for ECC measurements, because only two eyes showed ARP in ECC measurements.

In SLP-VCC measurements, TSS was not significantly correlated with age in simple correlation analysis
Table 1 Averages of the parameters of SLP with VCC and those with ECC in 105 normal and 82 OAG eyes

\begin{tabular}{|c|c|c|}
\hline & Normal & $O A G$ \\
\hline No. eyes & 105 & 82 \\
\hline \multicolumn{3}{|c|}{ Typical scan score } \\
\hline VCC & $90.4 \pm 16.4$ & $89.7 \pm 17.6$ \\
\hline ECC & $97.5 \pm 6.8$ & $98.4 \pm 4.6$ \\
\hline$P^{\mathrm{a}}$ & $<0.001$ & $<0.001$ \\
\hline \multicolumn{3}{|c|}{ TSNIT average } \\
\hline VCC & $55.6 \pm 5.3$ & $47.0 \pm 6.8$ \\
\hline ECC & $54.2 \pm 5.1$ & $45.5 \pm 5.6$ \\
\hline$P^{\mathrm{a}}$ & $<0.001$ & 0.011 \\
\hline \multicolumn{3}{|c|}{ Superior average } \\
\hline VCC & $68.4 \pm 7.7$ & $57.1 \pm 9.0$ \\
\hline ECC & $67.3 \pm 7.4$ & $56.7 \pm 8.8$ \\
\hline$P^{\mathrm{a}}$ & 0.009 & 0.598 \\
\hline \multicolumn{3}{|c|}{ Inferior average } \\
\hline VCC & $64.7 \pm 8.0$ & $52.3 \pm 10.8$ \\
\hline ECC & $67.9 \pm 7.6$ & $54.9 \pm 10.1$ \\
\hline$P^{\mathrm{a}}$ & $<0.001$ & $<0.001$ \\
\hline \multicolumn{3}{|c|}{ TSNIT SD } \\
\hline VCC & $22.8 \pm 4.5$ & $18.0 \pm 4.7$ \\
\hline ECC & $25.8 \pm 3.5$ & $20.7 \pm 4.2$ \\
\hline$P^{\mathrm{a}}$ & $<0.001$ & $<0.001$ \\
\hline \multicolumn{3}{|c|}{ Nerve fibre index } \\
\hline VCC & $18.9 \pm 8.5$ & $38.4 \pm 17.2$ \\
\hline ECC & $17.6 \pm 7.4$ & $36.0 \pm 15.3$ \\
\hline$P^{\mathrm{a}}$ & 0.036 & 0.064 \\
\hline
\end{tabular}

ECC $=$ enhanced corneal compensation; Normal $=$ ophthalmologically healthy eyes, $\mathrm{OAG}=$ open-angle glaucoma; $\mathrm{SLP}=$ scanning laser polarimetry; VCC $=$ variable corneal compensation.

Data are shown as mean $\pm \mathrm{SD}$.

${ }^{a} P$-value between the measurements with SLP with VCC and those with ECC (paired $t$-test).

(Pearson's correlation coefficient, $R=-0.12, P=0.104$ ), whereas the correlation became significant after adjusting for refractive error (partial correlation coefficient $(P C C)=-0.22, P=0.003$ ). TSS was significantly correlated with refractive error in simple correlation analysis $(R=0.19, P=0.011)$ and after adjusting for age $(\mathrm{PCC}=0.26, P<0.001)$. In the $82 \mathrm{OAG}$ eyes, MD was significantly correlated with TSS $(R=0.40$, $P<0.001$ ), and this correlation was still significant after adjusting for age and refractive error $(\mathrm{PCC}=0.35$, $P=0.001$ ).

In SLP-ECC measurements, TSS was significantly correlated with neither age nor refractive error in simple correlation analysis $(R=0.02, P=0.756 ; R=0.10$, $P=0.173$, respectively) and after adjusting for each other of age and refractive error ( $\mathrm{PCC}=-0.02, P=0.788$; $\mathrm{PCC}=0.10, P=0.177$, respectively). In the 82 OAG eyes, MD was significantly correlated with TSS in simple 
Table 2 Prevalence of ARP in measurements of SLP with VCC and that with ECC in 105 normal and 82 OAG eyes

\begin{tabular}{|c|c|c|c|c|c|c|c|}
\hline & \multicolumn{3}{|c|}{ Normal $(\mathrm{N}=105)$} & \multicolumn{3}{|c|}{$O A G(\mathrm{~N}=82)$} & \multirow[t]{2}{*}{${ }^{\mathrm{b}} \mathrm{P}$-value } \\
\hline & $\mathrm{N}$ & Prevalence & aP-value & $\mathrm{N}$ & Prevalence & aP-value & \\
\hline VCC & $14 / 105$ & $13.3(8.1-21.1) \%$ & 0.009 & $12 / 82$ & $14.6(8.6-23.9) \%$ & 0.002 & 0.834 \\
\hline ECC & 3/105 & $2.9(1.0-8.1) \%$ & & $1 / 82$ & $1.2(0.2-6.6) \%$ & & 0.632 \\
\hline
\end{tabular}

$\mathrm{ARP}=$ atypical retardation pattern; $\mathrm{ECC}=$ enhanced corneal compensation; Normal $=$ ophthalmologically healthy eyes, $\mathrm{OAG}=$ eyes with open-angle glaucoma; $\mathrm{SLP}=$ scanning laser polarimetry; $\mathrm{VCC}=$ variable corneal compensation.

The numbers of eyes with ARP and the prevalence (95\% confidence interval) are shown.

${ }^{\text {a }}$-value between the measurements with SLP-VCC and those with ECC (Fisher's exact test).

${ }^{\mathrm{b}} P$-value between the normal and OAG groups (Fisher exact test).


Figure 1 Correlation between RNFL average determined by scanning laser polarimetry with variable corneal compensation and mean deviation of Humphrey perimeter. (a) The correlation was not significant in all 82 open-angle glaucoma eyes (Pearson's correlation coefficient, $R=0.10, P=0.376$ ), (b) whereas it became significant after excluding the 12 eyes with atypical retardation pattern $(R=0.424, P<0.001)$.

correlation analysis $(R=0.23, P=0.038)$ and after adjusting for age and refractive error $(\mathrm{PCC}=0.23$, $P=0.039)$.
Correlation between MD of HFA and TSNIT average determined by SLP-VCC and -ECC were analysed in the 82 OAG eyes. Regarding VCC measurements, MD and TSNIT average were not significantly correlated in all 82 OAG eyes $(R=0.10, P=0.376$, Figure $1 \mathrm{a})$. However, when the 12 eyes showing ARP (ie, TSS $<80$ ) were excluded, the correlation became significant $(R=0.42$, $P<0.001$, Figure 1b), of which correlation coefficients were significantly higher than that in all 82 OAG eyes $(P=0.033)$. Regarding ECC measurements, MD and TSNIT average was significantly correlated with each other in all 82 eyes without excluding the eyes with ARP $(R=0.48, P<0.001$, Figure 2$)$.

\section{Discussion}

In this study, the prevalence of ARP, which was defined as an image with TSS less than 80, was estimated as 13.3 and $14.6 \%$ in VCC measurements in normal and OAG eyes, respectively. In an earlier study, Bagga et $a l^{8}$ found ARP in VCC measurements in $5(25 \%)$ of 20 normal and $23(51 \%)$ of 45 glaucomatous eyes. They subjectively determined ARP, which was defined as retardation maps with alternating peripapillary circumferential bands of low and high retardation, variable areas of high retardation arranged in a spokelike peripapillary pattern, or splotchy areas of nasal and temporal high retardation. Because severity of glaucoma was correlated with TSS as shown in this study, the difference in MD of glaucoma patients between Bagga's study ${ }^{8}$ and the current one $(-8.6 \pm 9.8$ vs $-3.3 \pm 4.1 \mathrm{~dB})$ should be one possible explanation for the discrepancy in the prevalence of ARP in glaucomatous eyes. More recently, the prevalence of ARP, defined by TSS $<80$, in VCC measurements in normal and glaucomatous eyes were reported as 10.4 and $15.5 \%$, respectively, by Toth et al; ${ }^{9}$ and 12.3 and $13.8 \%$, respectively, by Sehi et al, ${ }^{11}$ both of which were close to the current results. Gunvant et al ${ }^{16}$ reported relatively higher prevalence of ARP (21.2 and 22.3\% in normal and glaucomatous eyes, respectively). Although care should be taken because these prevalence data including ours 


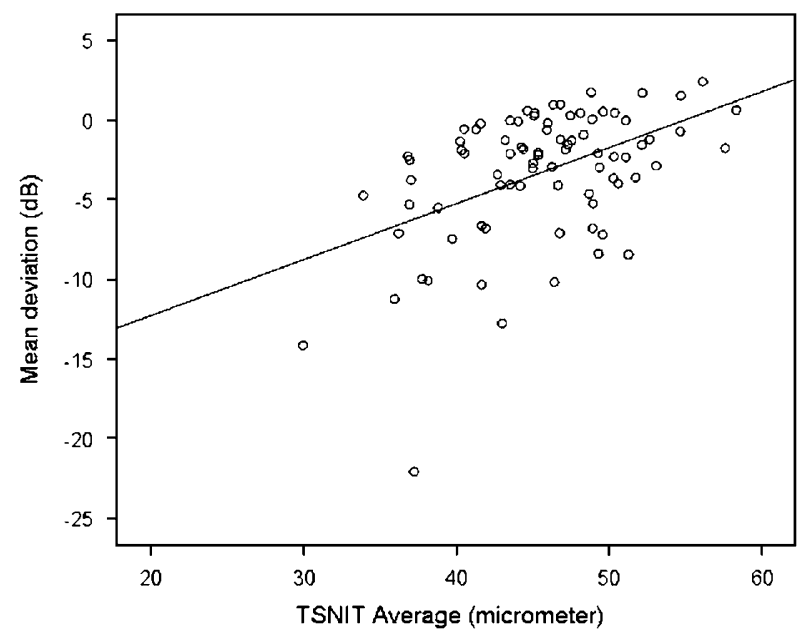

Figure 2 Correlation between RNFL average determined by scanning laser polarimetry with enhanced corneal compensation and mean deviation (MD) of Humphrey perimeter. The correlation was significant in all 82 open-angle glaucoma eyes (Pearson's correlation coefficient, $R=0.48, P<0.001$ ).

were based on clinical settings, not on population or epidemiological settings, the findings that more than $10 \%$ of normal or glaucomatous eyes show ARP in VCC measurements suggest the clinical limitation of VCC measurements as accurate evaluation of RNFL thickness is difficult in SLP results with ARP as shown in this study and in earlier ones. ${ }^{12,14}$

This study showed that the prevalence of ARP in ECC measurements were 2.9 and $1.2 \%$ in normal and OAG eyes, which was significantly lower than that in VCC measurements. The prevalence of ARP in ECC measurements was reported as 3.3 and $0 \%$ in 41 normal and 92 glaucomatous eyes, respectively, by Mai et $a l^{13}$ and $0.8 \%$ in 129 normal or glaucomatous eyes by Sehi et $a l^{11}$ These results including ours suggest that the ECC algorithm should improve the performance of SLP in RNFL thickness measurements, resulting in more accurate diagnosis of glaucoma ${ }^{12}$ and better correlation with visual field defects in glaucoma patients. ${ }^{14}$

Though the importance of ARP and TSS in SLP measurements has been widely recognized, the factors relating to them have not been fully understood. Only one earlier study has reported that TSS (ie, support vector machine score) was significantly associated with age, but not with refractive error or the amount of visual field damage. ${ }^{8}$ In this study, TSS was lower in older subjects or more myopic eyes after adjusting for each other. Because RNFL thickness is thinner in older than in younger subjects, ${ }^{21-23}$ signal-to-noise ratio in SLP measurements is easily reduced, resulting in reduction in TSS and higher prevalence of ARP. Although it is still debated whether RNFL thickness is thinner ${ }^{21}$ or not $^{24}$ in moderately myopic eyes compared to emmetropic eyes, RNFL thickness may partially influence the correlation between TSS and myopia. Hypopigmented fundus is often seen in myopic eyes. Noises from the scleral surface can be greater than usual in SLP measurements of such eyes, resulting in decrease in TSS, which should be a more probable explanation for the significant correlation between TSS and myopia found in this study. In ECC measurements, significant association of TSS and age or refractive error was not seen, suggesting better performance of ECC compared with VCC especially in older subjects or myopic eyes.

In the present OAG eyes, TSS in VCC measurements was also significantly correlated with MD of visual field results. It should be one possible explanation that OAG eyes with more advanced visual field damage had thinner RNFL, resulting in decreased signal-to-noise ratio in SLP measurements. Significant correlation between TSS and MD was found even in ECC measurements, although the correlation was a slightly weaker than in VCC. These findings in this study imply that the accuracy of RNFL thickness measurement using SLP-VCC/ECC may be reduced in advanced glaucomatous eyes.

We also evaluated the correlation between $\mathrm{MD}$ and RNFL thickness determined with SLP-VCC and -ECC in the 82 OAG eyes. Initially, the correlation was not significant in VCC measurements $(R=0.10, P=0.376)$. However, after excluding the 12 eyes with ARP, the correlation became significant $(R=0.42, P<0.001)$, suggesting that ARP disturbed accurate measurement of RNFL thickness with SLP. When SLP-VCC is used for the diagnosis or evaluation of glaucoma in clinical practice, eyes with ARP should be excluded or should be interpreted with caution. However, TSS is only available as an exportable value from the apparatus, and is not listed on the usual printout. Therefore, it is difficult to routinely exclude eyes with ARP according to TSS.

In conclusion, the current results suggest that ARP in VCC measurements was found in more than $10 \%$ of normal or glaucomatous eyes, and TSS in VCC measurements was significantly lower in older subjects, more myopic eyes, or eyes with more advanced glaucomatous damage. Moreover, the presence of ARP should disturb the accuracy of RNFL thickness measurement by SLP-VCC. In ECC measurements, prevalence of ARP was considerably lower compared with VCC measurements, and TSS was significantly correlated only with the extent of glaucomatous visual field damage but not with age or refractive error. Therefore, we suggest that SLP-VCC scans with ARP should be interpreted with caution in clinical practice or should be excluded from a clinical study. On the other hand, ECC measurements should decrease the frequency 
of scans with ARP even in older subjects or myopic eyes, resulting in the improvement of performance of SLP for diagnosis and evaluation of glaucoma in clinical practices.

\section{References}

1 Quigley HA, Green WR. The histology of human glaucoma cupping and optic nerve damage: clinicopathologic correlation in 21 eyes. Ophthalmology 1979; 86: 1803-1830.

2 Quigley HA, Miller NR, George T. Clinical evaluation of nerve fiber layer atrophy as an indicator of glaucomatous optic nerve damage. Arch Ophthalmol 1980; 98: 1564-1571.

3 Quigley HA, Addicks EM, Green WR. Optic nerve damage in human glaucoma. III. Quantitative correlation of nerve fiber loss and visual field defect in glaucoma, ischemic neuropathy, papilledema, and toxic neuropathy. Arch Ophthalmol 1982; 100: 135-146.

4 Sommer A, Katz J, Quigley HA, Miller NR, Robin AL, Richter RC et al. Clinically detectable nerve fiber atrophy precedes the onset of glaucomatous field loss. Arch Ophthalmol 1991; 109: 77-83.

5 Weinreb RN, Shakiba S, Sample PA, Shahrokni S, van Horn $\mathrm{S}$, Garden VS et al. Association between quantitative nerve fiber layer measurement and visual field loss in glaucoma. Am J Ophthalmol 1995; 120: 732-738.

6 Hoh ST, Greenfield DS, Liebmann JM, Maw R, Ishikawa H, Chew $\mathrm{SJ}$ et al. Factors affecting image acquisition during scanning laser polarimetry. Ophthalmic Surg Lasers 1998; 29: 545-551.

7 Bozkurt B, Irkec M, Gedik S, Orhan M, Erdener U, Tatlipinar $\mathrm{S}$ et al. Effect of peripapillary chorioretinal atrophy on GDx parametersin patients with degenerative myopia. Clin Experiment Ophthalmol 2002; 30: 411-414.

8 Bagga H, Greenfield DS, Feuer WJ. Quantitative assessment of atypical birefringence images using scanning laser polarimetry with variable corneal compensation. Am J Ophthalmol 2005; 139: 437-446.

9 Toth M, Hollo G. Enhanced corneal compensation for scanning laser polarimetry on eyes with atypical polarisation pattern. Br J Ophthalmol 2005; 89: 1139-1142.

10 Sehi M, Guaqueta DC, Greenfield DS. An enhancement module to improve the atypical birefringence pattern using scanning laser polarimetry with variable corneal compensation. Br J Ophthalmol 2006; 90: 749-753.

11 Sehi M, Guaqueta DC, Feuer WJ, Greenfield DS. Scanning laser polarimetry with variable and enhanced corneal compensation in normal and glaucomatous eyes. Am J Ophthalmol 2007; 143: 272-279.

12 Bowd C, Medeiros FA, Weinreb RN, Zangwill LM. The effect of atypical birefringence patterns on glaucoma detection using scanning laser polarimetry with variable corneal compensation. Invest Ophthalmol Vis Sci 2007; 48: 223-227.

13 Mai TA, Reus NJ, Lemij HG. Diagnostic accuracy of scanning laser polarimetry with enhanced versus variable corneal compensation. Ophthalmology 2007; 114: 1988-1993.

14 Saito H, Tomidokoro A, Yanagisawa M, Aihara M, Tomita G, Araie M. Scanning laser polarimetry with enhanced corneal compensation in patients with open-angle glaucoma. J Glaucoma 2008; 17: 24-29.

15 Monteiro ML, Moura FC, Medeiros FA. Scanning laser polarimetry with enhanced corneal compensation for detection of axonal loss in band atrophy of the optic nerve. Am J Ophthalmol 2008; 145: 747-754.

16 Gunvant P, Zheng Y, Toth M, Hollo G. Atypical retardation pattern: can performance of classification be improved? Optom Vis Sci 2008; 85: 482-488.

17 Knighton RW, Zhou Q. New techniques. In: Iester M, Garway-Heath D, Lemij HG (eds). Optic Nerve Head and Retinal Nerve Fibre Analysis. Dogma: Savona, Italy, 2005, pp 117-119.

18 Toth M, Hollo G. Evaluation of enhanced corneal compensation in scanning laser polarimetry: comparison with variable corneal compensation on human eyes undergoing LASIK. J Glaucoma 2006; 15: 53-59.

19 Bagga H, Greenfield DS. Quantitative assessment of structural damage in eyes with localized visual field abnormalities. Am J Ophthalmol 2004; 137: 797-805.

20 Anderson DR, Pattela VM. Automated Static Perimetry. Mosby: St Louis, 1999, pp 152-153.

21 Budenz DL, Anderson DR, Varma R, Schuman J, Cantor L, Savell J et al. Determinants of normal retinal nerve fiber layer thickness measured by Stratus OCT. Ophthalmology 2007; 114: 1046-1052.

22 Tjon-Fo-Sang MJ, de Vries J, Lemij HG. Measurement by nerve fiber analyzer of retinal nerve fiber layer thickness in normal subjects and patients with ocular hypertension. Am J Ophthalmol 1996; 122: 220-227.

23 Poinoosawmy D, Fontana L, Wu JX, Fitzke FW, Hitchings RA. Variation of nerve fibre layer thickness measurements with age and ethnicity by scanning laser polarimetry. $\mathrm{Br} J$ Ophthalmol 1997; 81: 350-354.

24 Hoh ST, Ishikawa H, Greenfield DS, Liebmann JM, Chew SJ, Ritch R. Peripapillary nerve fiber layer thickness measurement reproducibility using scanning laser polarimetry. J Glaucoma 1998; 7: 12-15. 